\title{
Efeito do entrelaçador na comparação de desempenho de sistemas OFDM e SCCP
}

\author{
Amanda de Paula e Cristiano Panazio
}

\begin{abstract}
Resumo-Este artigo efetua uma comparação entre os sistemas OFDM (Orthogonal Frequency Division Multiplexing) e SCCP (Single Carrier with Cyclic Prefix) do ponto de vista do entrelaçador. É realizada uma análise através de simulações do impacto da estrutura de entrelaçamento nos sistemas, em canais seletivos em frequência com desvanecimento por blocos e estáticos.
\end{abstract}

Palavras-Chave-OFDM, SCCP, entrelaçamento, canais seletivos em frequência.

Abstract-This article establishes a performance comparison between the OFDM (Orthogonal Frequency Division Multiplexing) and the SCCP (Single Carrier with Cyclic Prefix) systems from the interleaver point of view. Some interleavers schemes are analyzed in a block fading channel scenario, as well as in the context of deep fade static channels.

Keywords-OFDM, SCCP, interleaving, frequency selective channels.

\section{INTRODUÇÃO}

O sistema OFDM é uma técnica de transmissão de dados bastante popular que consiste em transmitir sinais digitais através de um conjunto de subportadoras ortogonais. Uma das grandes vantagens envolvendo a transmissão OFDM é a possibilidade de equalizar o sinal através de uma simples correção de ganho e de fase (one-tap equalizer), desde que um prefixo cíclico ( $\mathrm{CP}$, do inglês, cyclic prefix), de tamanho adequado, seja adicionado ao sinal transmitido.

Todavia, caso o canal não seja conhecido na transmissão, o desempenho do OFDM é bem mais sensível a forma de codificação de canal adotada, pois dela depende a recuperação da informação perdida em subportadoras que sejam fortemente atenuadas [1], [2]. Admitindo que não é sempre possível dispor do conhecimento do canal na transmissão, um modo de aumentar a robustez neste caso é através da utilização de pré-codificação linear entre os símbolos a serem transmitidos em cada subportadora [3], [4]. Esta técnica permite espalhar a informação pelas subportadoras, de modo que seja mais simples a recuperação da mesma caso algumas subportadoras estejam desvanecidas. Uma forma bastante interessante de realizar tal pré-codificação é a utilização da transformada discreta de Fourier. Neste caso particular, o OFDM precodificado é equivalente a uma transmissão por portadora única. Como o OFDM utiliza CP, denomina-se tal técnica de SCCP (Single Carrier with Cyclic Prefix), cuja equalização também é realizada através do one-tap equalizer, i.e., com uma complexidade equivalente àquela do OFDM.

Amanda de Paula e Cristiano Panazio, Escola Politécnica, Universidade de São Paulo, São Paulo, Brasil, E-mails: amanda@lcs.poli.usp.br, cpanazio@1cs.poli.usp.br.
Tal forma de transmissão tem despertado o interesse da comunidade e várias comparações entre OFDM e SCCP tem sido apresentadas. Em [3] foi demonstrado que, no caso sem codificação de canal e modulação QPSK (Quaternary Phase Shift Keying), o desempenho do SCCP equalizado com filtro linear é superior ao do OFDM para qualquer condição de canal. A generalização da comparação para o caso de transmissão utilizando códigos corretores de erros não é uma tarefa simples. Em [2] é feita uma análise baseada na cutoff rate, o que pressupõe códigos aleatórios e de comprimento infinito. Nesse trabalho é mostrado que ambas as técnicas possuem desempenho equivalente para modulação QPSK e rendimento menor ou igual a 0,5. Para rendimentos maiores, o SCCP se mostra mais robusto, o que corrobora com [3]. Já em [5] é mostrado que ambos os sistemas, devidamente codificados, conseguem alcançar a diversidade em frequência do canal. Em [1], [6], [7], [8] e [9] são mostrados alguns resultados baseados em simulação envolvendo questões como taxa de codificação e PAPR (Peak to Average Power Rate) .

Neste contexto, uma importante questão ainda pouco abordada na literatura é a robustez das técnicas em relação a maneira de entrelaçar os bits codificados. Visando contribuir para a minimização dessa lacuna, no presente trabalho, compararemos os sistemas em diversas condições de entrelaçamento e apontaremos quais sistemas apresentam maior robustez.

Este trabalho encontra-se organizado como se segue. $\mathrm{Na}$ seção 2 apresentamos o modelo universal adotado para os sistemas SCCP e OFDM. Na seção 3, abordamos a questão do projeto do entrelaçador e na seção 4 prosseguimos com uma comparação de desempenho entre os sistemas. Finalmente, indicamos as conclusões do nosso trabalho na seção 5 .

\section{Modelo do SistemA}

O modelo do sistema está apresentado na Fig. 1. A matriz de pré-codificação linear $\mathbf{P}$ é o único fator que distingue as duas técnicas. No caso OFDM, como o sinal é gerado através da aplicação da IFFT (Inverse Fast Fourier Transform) na sequência de símbolos, a matriz $\mathbf{P}$ deve ser substituída pela matriz identidade. Já no contexto SCCP, a própria sequência de símbolos é transmitida, assim a matriz de pré-codificação linear deve ser substituída pela matriz de Fourier.

O sinal com prefixo cíclico é transmitido por um canal de resposta impulsiva $\{h(n)\}$ e ruído branco é adicionado. No receptor, o prefixo cíclico é descartado e a equalização se dá no domínio da frequência com um one-tap equalizer de coeficientes $W(k)$.

Esses coeficientes de equalização $W(k)$ podem ser obtidos de diferentes maneiras. Os dois critérios clássicos de 


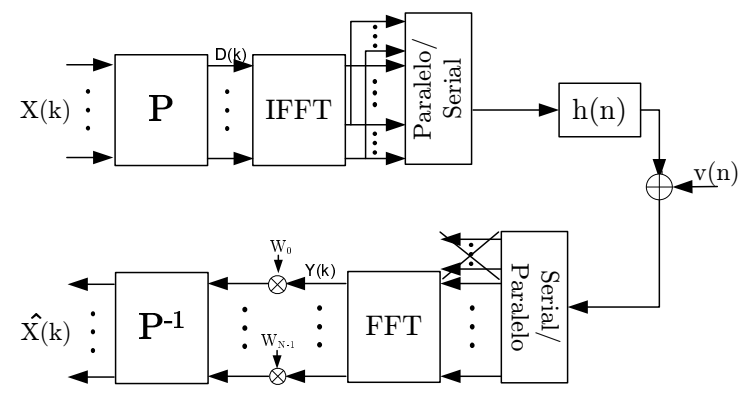

Fig. 1. Abordagem universal

equalização são o zero-forcing (ZF) e critério de mínimo erro médio quadrático (MMSE).

No caso OFDM, o critério de equalização não modifica a SNR (Signal to Noise Rate) por bit na saída do equalizador. A única função do equalizador é corrigir a magnitude e principalmente a fase do sinal.

Já no caso SCCP, a presença de interferência intersimbólica (ISI) faz com que seja necessário especial cuidado na escolha do critério de equalização. O critério ZF elimina a ISI, desde que o canal não apresente nulos espectrais, mas pode gerar uma amplificação de ruído extremamente elevada em canais com desvanecimentos profundos. Já o critério MMSE, busca um compromisso entre a eliminação da ISI e a minimização da amplificação do ruído. O desempenho do equalizador MMSE supera o do equalizador ZF e, por tal motivo, iremos adotar esse critério de equalização, no caso do SCCP.

Denotando o vetor de coeficientes do equalizador por $\mathbf{W}$, o vetor de sinais desejados por $\mathbf{D}=\mathbf{P X}$ e o vetor recebido por $\mathbf{Y}$, o critério de equalização MMSE é dado pela seguinte expressão:

$$
\underset{\mathbf{W}}{\operatorname{argmin}} \mathbb{E}\left\{\|\mathbf{D}-\mathbf{W} \circ \mathbf{Y}\|^{2}\right\}
$$

Na expressão acima, o operador $\{\cdot\} \circ\{\cdot\}$ corresponde ao produto de Hadamard (produto termo a termo).

O problema de otimização apresentado na eq. (1) apresenta um único mínimo, o qual é obtido quando os coeficientes de equalização são dados por:

$$
W_{M M S E}(k)=\frac{\sigma_{X}^{2} H^{*}(k)}{\sigma_{X}^{2}|H(k)|^{2}+\sigma_{v}^{2}} \quad k=0, \cdots, N-1
$$

em que $\sigma_{X}^{2}$ é a potência do sinal transmitido, $\sigma_{v}^{2}$ é a potência do ruído na recepçã, $H(k)$ é a $k$-ésima componente em frequência do canal. Os coeficientes na eq. (2) foram derivados assumindo que a matriz $\mathbf{P}$ é unitária.

A modulação SCCP ainda pode ser equalizada com o DFE (Decision Feedback Equalizer) implementado no domínio da frequência. Neste caso, os coeficientes são derivados como mostrado em [8].

Em relação à codificação de canal, podemos implementála através de um TCM (Trellis Coded Modulation) [10] ou através de um BICM (Bit Interleaved Coded Modulation) [11]. Em [11] é mostrado que, para canais variantes no tempo, essa estrutura de codificação é mais adequada do que o TCM. Como em canais seletivos em frequência os símbolos recebidos nas subportadoras podem ser vistos como se tivessem sido submetidos a um canal variante no tempo, a técnica BICM parece ser a mais adequada. Adotaremos essa

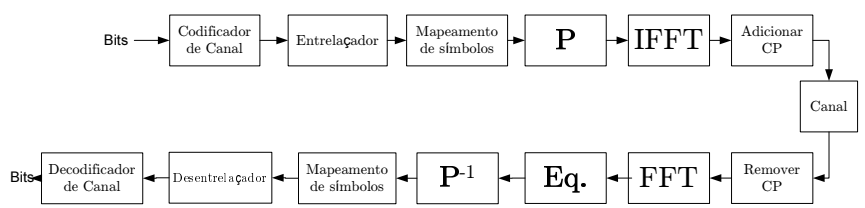

Fig. 2. Modelo do sistema com codificação

estrutura de codificação e o modelo global do sistema adotado é representado pelo diagrama na Fig. 2.

\section{Projeto Do EntrelaçAdor}

Os entrelaçadores são componentes fundamentais dos sistemas de codificação. Sua principal aplicação reside em evitar rajada de erros em códigos projetados para o canal com ruído branco e gaussiano (AWGN, em inglês), em que os erros ocorrem de forma descorrelacionada. No caso do OFDM, rajadas de erros podem ocorrer quando um conjunto contíguo de subportadoras apresenta forte desvanecimento. Já no contexto SCCP, o equalizador geralmente correlaciona o ruído que se soma aos símbolos equalizados, podendo gerar assim uma rajada de erros.

O entrelaçamento nada mais é do que uma função de permutação aplicada a uma sequência de bits. Há basicamente dois tipos de entrelaçadores: os entrelaçadores de bloco e os entrelaçadores convolucionais. Nos detendo nos entrelaçadores de bloco, ainda temos várias maneiras de definir a permutação. Analisaremos o entrelaçador regular, o entrelaçador modular, apresentado em [12], e o entrelaçador S-aleatório [13].

O entrelaçador regular é a maneira clássica de entrelaçar bits. Para entrelaçar $N_{b}$ bits, define-se uma matriz de entrelaçamento com $m$ linhas e $n=N_{b} / m$ colunas. O procedimento de entrelaçamento de um vetor $\mathbf{x}$ de bits consiste em preencher a matriz por suas linhas e ler os bits através das colunas como ilustrado na expressão abaixo:

$$
\begin{gathered}
{\left[\begin{array}{c}
x_{1} \\
x_{2} \\
x_{3} \\
\vdots \\
x_{N_{b}}
\end{array}\right] \stackrel{\text { escrita }}{\longrightarrow}\left[\begin{array}{cccc}
x_{1} & x_{2} & \cdots & x_{n} \\
x_{n+1} & x_{n+2} & \cdots & x_{2 n} \\
\vdots & \vdots & & \vdots \\
x_{(m-1) n+1} & x_{(m-1) n+2} & \cdots & x_{N_{b}}
\end{array}\right]} \\
\text { leitura } \\
{\left[\begin{array}{c}
x_{1} \\
x_{n+1} \\
x_{2 n+1} \\
\vdots \\
x_{N_{b}}
\end{array}\right]}
\end{gathered}
$$

Uma outra forma de entrelaçar os bits do vetor $\mathbf{x}$ é através de um entrelaçador modular. Denotando por $\mathbf{k}$ o conjunto de índices do vetor $\mathbf{x}$, o entrelaçador modular é implementado aplicando a seguinte função de permutação à sequência de índices original:

$$
\Pi(\mathbf{k}) \equiv N_{1} \mathbf{k} \bmod \left(N_{b}\right)
$$

em que $N_{1}$ é o parâmetro do entrelaçador, que pode ser escolhido como qualquer inteiro menor que $N_{b}$ desde que $\operatorname{MDC}\left(N_{1}, N_{b}\right)=1$. Essa última condição garante a injetividade do mapeamento de índices. Nessa situação, existe o 


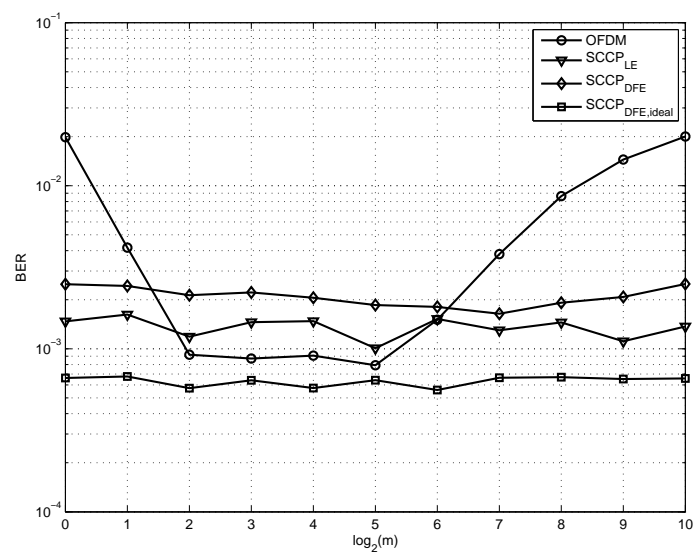

Fig. 3. Efeito da configuração do entrelaçador regular no canal com função transferência dada pela eq. (5), $E_{b} / N_{o}=12 \mathrm{~dB}$

inteiro $N_{1}^{-1}$, tal que $N_{1} N_{1}^{-1} \equiv 1 \bmod \left(N_{b}\right)$. A função de desentrelaçamento é dada por:

$$
\Pi^{-1}(\mathbf{k}) \equiv N_{1}^{-1} \mathbf{k} \quad \bmod \left(N_{b}\right)
$$

Finalmente, o entrelaçador S-aleatório é obtido a partir de qualquer permutação aleatória da sequência de bits, de modo que seja garantida uma distância mínima $d_{\text {min }}=S$ entre quaisquer $S$ bits consecutivos da sequência original [13].

A seguir, será feita uma análise de desempenho dos sistemas em função do tipo de entrelaçador escolhido. Iremos segmentar a análise em dois casos distintos: canais seletivos em frequência com desvanecimento Rayleigh por blocos e canais seletivos em frequência estáticos.

Em ambos os casos iremos assumir modulação QPSK, código corretor de erros com taxa $R=1 / 2$ e blocos de $N_{s}=512$ símbolos ou subportadoras. O entrelaçamento será realizado permutando os bits de um mesmo bloco. No contexto de canais com desvanecimento, utilizaremos o código de polinômio gerador $\left[\begin{array}{lll}133 & 171\end{array}\right]_{\text {octal }}$. Já no caso de canais estáticos, será utilizado o código de polinômio gerador $\left[\begin{array}{ll}15 & 17\end{array}\right]_{\text {octal }}$.

Os coeficientes do equalizador são obtidos a partir do conhecimento perfeito do canal e da variância do ruído.

\section{A. Canais seletivos em frequência com desvanecimento Ray- leigh por blocos}

A configuração do canal escolhido nesse contexto é dada por:

$$
H(z)=h_{0}+h_{1} z^{-1}+h_{2} z^{-2}
$$

em que $h_{k}$ são variáveis gaussianas complexas com variância $\sigma_{h}^{2}=1 / 3$.

Analisaremos como os sistemas se comportam em função das possíveis escolhas de estrutura de entrelaçamento. Essa análise será feita fixando-se a relação $E_{b} / N_{o}=12 \mathrm{~dB}$ e observando a BER (Bit Error Rate) gerada pelos sistemas para os diferentes entrelaçadores.

Nos atendo inicialmente ao caso de entrelaçamento regular, analisaremos o impacto da escolha do número de linhas do entrelaçador no desempenho do sistema. Os resultados estão expostos na Fig. 3.

As curvas na Fig. 3 indicam que o sistema OFDM apresenta uma sensibilidade muito maior em relação à escolha do

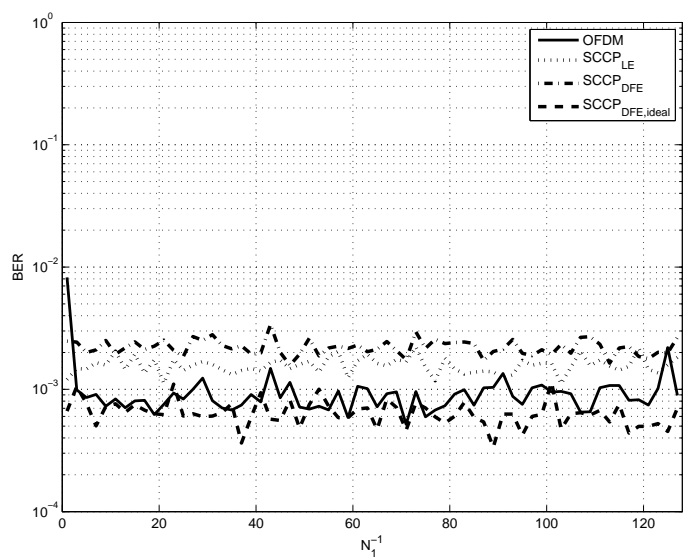

Fig. 4. BER em função do parâmetro $N_{1}^{-1}$ do entrelaçador modular, canal com desvanecimento Rayleigh e relação $E_{b} / N_{o}=12 \mathrm{~dB}$

entrelaçador do que o sistema SCCP. Não há flutuações significativas no desempenho do SCCP provocadas pela variação na configuração da matriz de entrelaçamento. No sistema OFDM, por sua vez, as variações apresentadas excedem uma década. É ainda importante observar que o desempenho do SCCP equalizado com o DFE é inferior ao equalizado com filtro linear. Esse fato pode ser atribuído ao fenômeno de propagação de erros inerente ao DFE. Caso sejam empregados mecanismos de minimização de propagação de erros, como o descrito em [14], o desempenho do DFE pode se aproximar do desempenho do DFE ideal, em que o processo de realimentação está livre de erros.

Em relação ao entrelaçador modular, obtemos a BER em função de $N_{1}^{-1}$, mostrada na Fig. 4. A fim de facilitar a visualização, os resultados indicados na Fig. 4 foram gerados a partir de blocos de $N_{s}=64$.

Analisando as curvas da Fig. 4 percebemos que, inclusive para o caso OFDM, a escolha do parâmetro de entrelaçamento modular não traz grandes impactos no desempenho do sistema. Também simulamos blocos com $N_{s}=512$ e o mesmo comportamento foi observado.

Nos resta analisar o desempenho do entrelaçador Saleatório. Para um parâmetro $S$ fixado, foram gerados cinquenta entrelaçadores e selecionado aquele que fornecia a menor BER. Os resultados obtidos para vinte e cinco mil realizações de canal estão mostrados na Fig. 5. Analisando os resultados, percebemos que, inclusive no sistema OFDM, o desempenho do sistema é invariante à escolha do parâmetro $S$ de entrelaçamento.

Comparando os resultados mostrados nas Fig. 3, Fig. 4 e Fig. 5, notamos que o entrelaçador S-aleatório apresenta os melhores resultados para o OFDM e a para o SCCP equalizado com o DFE. O desempenho do sistema SCCP equalizado com filtro linear é independente do tipo de entrelaçamento utilizado.

\section{B. Canais estáticos com desvanecimento profundo}

Iremos agora considerar canais estáticos com desvanecimentos em frequência profundos. Como primeiro exemplo, seja o canal Proakis B [15] com função transferência dada por:

$$
H(z)=0,407+0,815 z^{-1}+0,407 z^{-2}
$$




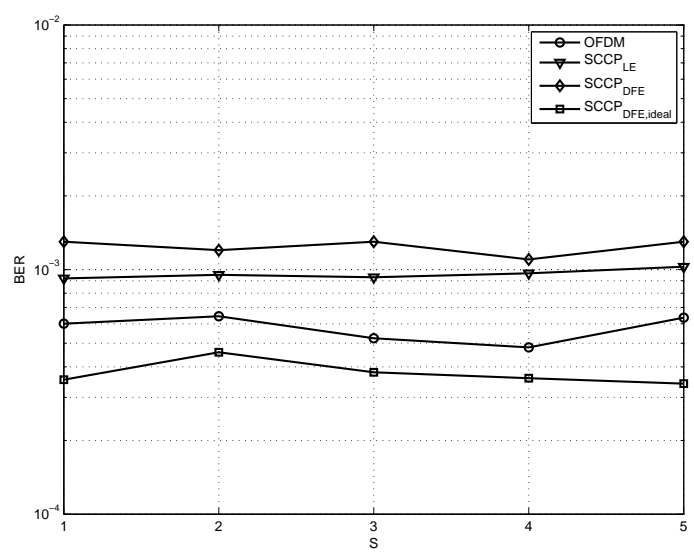

Fig. 5. BER em função do parâmetro $S$ do entrelaçador S-aleatório, canal com desvanecimento Rayleigh e relação $E_{b} / N_{o}=12 \mathrm{~dB}$

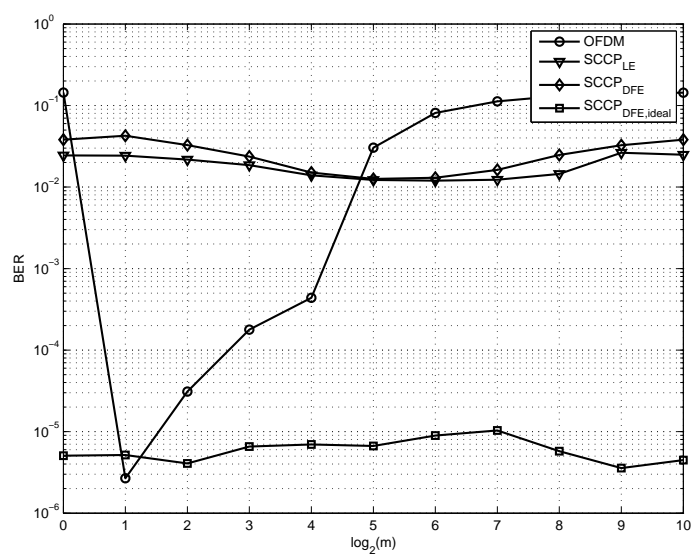

Fig. 6. BER para entrelaçadores regulares com diferentes números de linhas, para o canal Proakis B, $E_{b} / N_{o}=8 d B$

Assim como feito no caso de canais com desvanecimento, analisaremos como os sistemas se comportam para diferentes estruturas de entrelaçamento.

Quanto ao entrelaçador regular, a Fig. 6 mostra que a sensibilidade do sistema OFDM em relação à escolha do parâmetro de entrelaçamento é ainda mais evidente do que no caso de canais com desvanecimento por bloco. A diferença de desempenho do OFDM, em termos de BER, entre a melhor e a pior configuração de entrelaçamento excede quatro décadas.

O sistema SCCP é praticamente invariante em relação à configuração do entrelaçador regular utilizado. Aqui vemos mais uma vez, e de forma amplificada, a diferença entre o DFE e sua versão ideal. Novamente, também observamos que o desempenho do DFE é, na melhor configuração de entrelaçador, apenas equivalente ao SCCP com filtro linear. O fenômeno de propagação de erros explica tais resultados.

Para justificar a discrepância entre os resultados obtidos para o OFDM, lembramos que os símbolos são modulados com QPSK e que em cada bloco de $N_{s}$ símbolos há $N_{b}=$ $2 N_{s}$ bits. Considerando que os bits associados a um dado símbolo codificado têm a mesma SNR, a SNR do $k$-ésimo bit codificado é proporcional a $|H(\lfloor k / 2\rfloor)|^{2}$. O entrelaçamento será eficiente se as rajadas de erros forem evitadas, ou seja, se os bits em condições de SNR desfavoráveis estiverem intercalados com os bits em boas condições de SNR.

Como este canal é um filtro passa-baixa, os bits das posições intermediárias do bloco recebido devem estar em

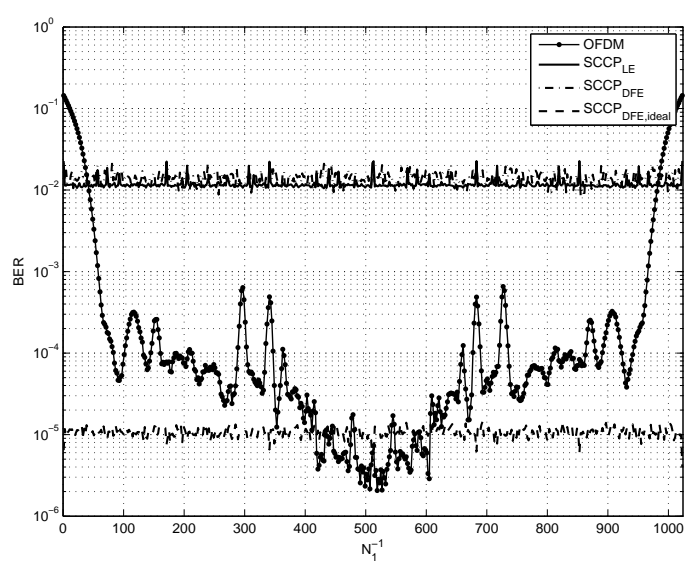

Fig. 7. BER em função do parâmentro $N_{1}^{-1}$ do entrelaçador modular, canal com função de transferência dada pela eq. (6) e relação $E_{b} / N_{o}=8 \mathrm{~dB}$

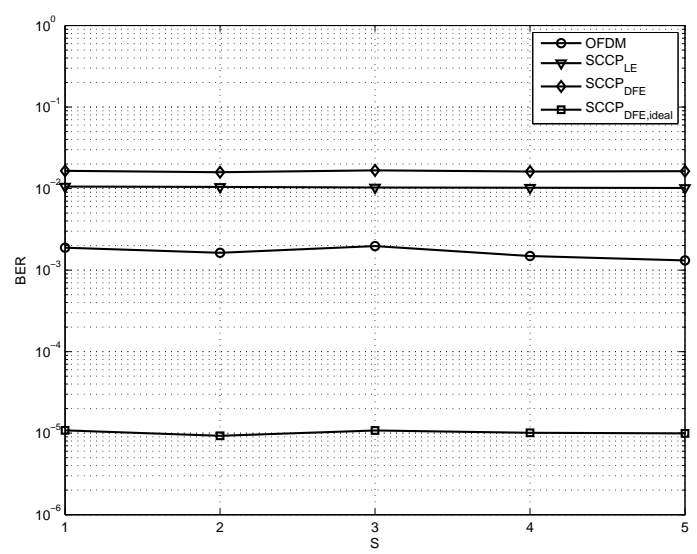

Fig. 8. BER em função do parâmetro $S$ para o canal com função transferência dada pela eq. (6), relação $E_{b} / N_{o}=8 \mathrm{~dB}$

condições de baixa SNR e os primeiros e os últimos bits devem apresentar SNR elevadas. Portanto, seria interessante que o entrelaçamento garantisse um espaçamento de $n=N_{b} / 2$ bits entre dois bits consecutivos do bloco. Para que essa condição seja atendida, a regra de mapeamento regular estabelecida implica na escolha de uma matriz de entrelaçamento com $n=$ $N_{b} / 2$ colunas e, consequentemente, $m=N_{b} / n=2$ linhas. Se essa condição não for obedecida, a probabiblidade de ocorrer rajadas de erros aumenta, comprometendo o desempenho do decodificador de canal no OFDM.

Vejamos se o entrelaçador modular também apresenta essa sensibilidade quanto ao parâmetro de entrelaçamento. A Fig. 7 mostra as flutuações na BER para diferentes valores do parâmetro $N_{1}^{-1}$ do entrelaçador modular. Os resultados apontam para uma forte dependência do sistema OFDM com o parâmetro de entrelaçamento $N_{1}$. Da mesma forma que no caso do entrelaçamento regular, o sistema SCCP apresenta robustez quanto ao parâmetro do entrelaçador modular.

Finalmente, vamos analisar o desempenho dos sistemas quando o entrelaçador implementado é do tipo S-aleatório.

A Fig. 8 mostra que todas as técnicas são robustas quanto à escolha do parâmetro $\mathrm{S}$. Quando comparamos esses resultados com aqueles mostrados nas Fig. 6 e Fig. 7, percebemos o entrelaçador S-aleatório não gera bons resultados para esse tipo de canal no contexto OFDM. Nesse caso, os entrelaçadores modular e regular levam aos melhores resultados. Para o entrelaçador regular, o número de linhas adequado 


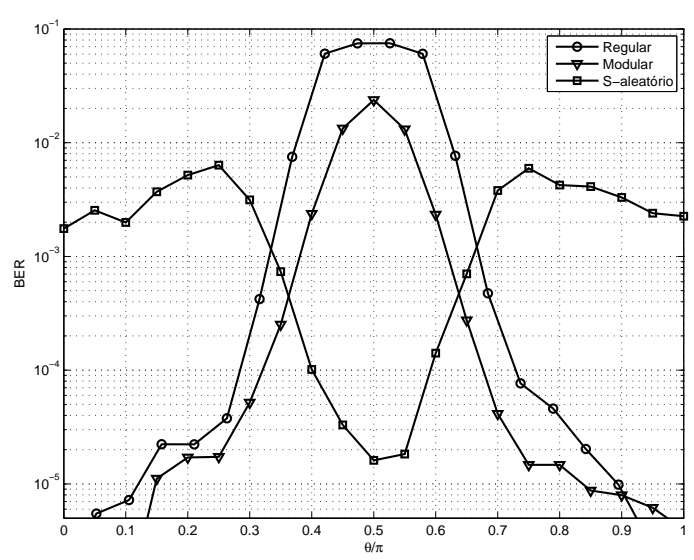

Fig. 9. Sistema OFDM: BER para entrelaçador regular com $m=4$ linhas, entrelaçador modular com $N_{1}=951$ e entrelaçado S-aleatório com $S=4$, para canais com função transferência expressas pela eq. (7), $E_{b} / N_{o}=8 d B$

é $m=2$. Para o entrelaçador modular, a Fig. 7 indica que a escolha mais adequada para o parâmetro de entrelaçamento seria $N_{1}^{-1}=519 \rightarrow N_{1}=(519)^{-1} \bmod (1024)=951$. Cabe ressaltar que as configurações $S=1$ e $S=2$ permitiriam a construção de entrelaçadores equivalentes aos melhores entrelaçadores regular e modular. Todavia, tal realização é improvável dado o comprimento do bloco e o número de realizações do entrelaçador $\mathrm{S}$-aleatório testadas.

A pergunta que surge é se, no contexto OFDM, esses entrelaçadores ainda geram bons resultados em outros tipos de canais, mantendo-se fixados os parâmetros obtidos para o caso do canal Proakis B. Para responder a essa pergunta, vamos definir a seguinte família de canais com nulo espectral:

$$
H(z)=\left(1-e^{j \theta} z^{-1}\right)\left(1-e^{-j \theta} z^{-1}\right) \quad 0 \leq \theta \leq \pi
$$

e fixar a relação $E_{b} / N_{o}=8 d B$. Como a escolha do parâmetro $S$ não traz grandes impactos no desemepenho do sistema, iremos arbitrariamente fixar $S=4$.

A Fig. 9 mostra que se o canal tiver característica passaalta ou passa-baixa, os entrelaçadores regular e modular são adequados. O desempenho do melhor entrelaçador modular é, inclusive, superior ao melhor entrelaçador regular para as situações de canal expressas pela eq. (7). Se o canal for do tipo rejeita-faixa, com zero em torno de $\theta=\pi / 2$, o desempenho do sistema é extremamente degradado com esses tipos de entrelaçadores. Nessas situações, o entrelaçador S-aleatório apresenta desempenho bem superior ao dos entrelaçadores regular e modular, apesar de mostrar piores resultados no contexto de canais passa-alta ou passa-baixa. Além disso, é importante enfatizar que a pior situação de canal para o entrelaçador S-aleatório gera uma degradação bem menor do que a provocada por canais pouco favoráveis ao entrelaçador modular e ao entrelaçador regular.

\section{COMPARAÇÃO DE DESEMPENHO OFDM X SCCP}

Nesta seção, selecionaremos configurações de entrelaçamento adequadas e compararemos os dois sistemas em termos de BER em função da $E_{b} / N_{o}$.

Primeiramente, verificaremos como os sistemas se comportam em canais seletivos em frequência com desvanecimento Rayleigh por blocos.

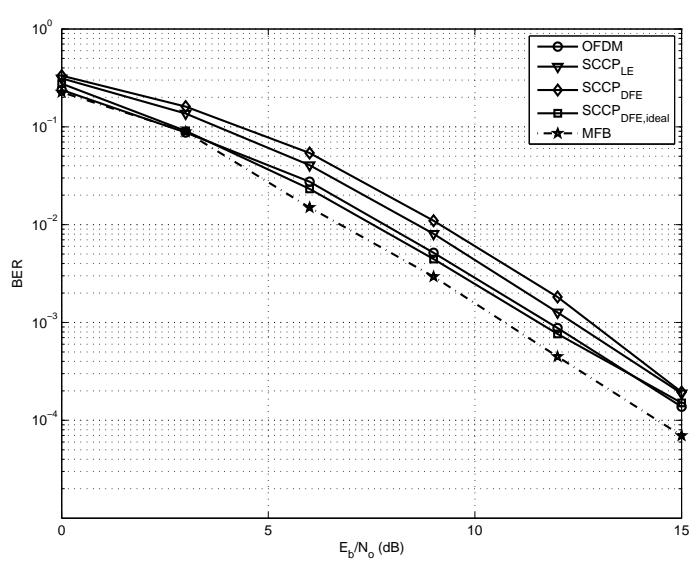

Fig. 10. Comparação de desempenho OFDM e SCCP em canais seletivos em frequência com desvanecimento Rayleigh. Entrelaçador S-aleatório com $S=4$.

$\mathrm{Na}$ seção anterior, vimos que, para essa configuração de canal, o entrelaçamento S-aleatório é o mais adequado para os casos OFDM e SCCP equalizado com DFE. Já para o SCCP equalizado com o filtro linear, as estruturas de entrelaçamento analisadas levavam ao mesmo desempenho. Dessa forma, adotaremos o entrelaçador S-aleatório em ambos os sistemas. Como o desempenho dos sistemas é invariante ao parâmentro $S$ neste contexto, iremos, arbitrariamete, impor $S=4$ na comparação que se segue.

Juntamente com as curvas de BER, exibiremos uma curva de MFB (Matched Filter Bound). Essa curva é um limitante que indica o desempenho de um sistema capaz de maximizar a SNR sem introduzir a interferência intersimbólica característica de um filtro casado.

A Fig. 10 mostra que no caso de canais seletivos em frequência com desvanecimento Rayleigh por bloco, o OFDM apresenta desempenho comparável ao DFE perfeito.

Voltando as atenções para o caso de canal estático com desvanecimento profundo, lembramos a grande perda de desempenho gerada no DFE pelo fenômeno de propagação de erros. Visando mitigar esse fato, empregaremos a técnica descrita em [14], na qual o DFE utiliza como realimentação decisões provenientes do decodificador de canal.

Considerando o canal Proakis B, a Fig. 11 mostra uma comparação de desempenho entre os sistemas. Para essa configuração de canal, os resultados da seção anterior mostram que para o contexto de SCCP, o entrelaçador regular com $m=32$ linhas seria adequado. Já para o OFDM, adotaremos o entrelaçador regular com $m=2$ linhas.

Analisando os resultados indicados na Fig.11, notamos que o desempenho do OFDM é comparável ao do DFE ideal, sendo bem superior ao do SCCP equalizado com filtro linear. Para relações $E_{b} / N_{o}$ mais elevadas, o desempenho do OFDM chega a superar o do DFE ideal. Além disso é importante destacar o considerável ganho de desempenho promovido pela aplicação do mecanismo de redução de propagação de erros no DFE.

Nessa configuração de canal específica, conseguimos projetar um entrelaçador de tal forma que o desempenho do OFDM fosse comparável com o do DFE ideal. É natural perguntar se para qualquer configuração de canal ainda seria possível projetar um entrelaçador que aproxime o desempenho do OFDM do DFE ideal. 


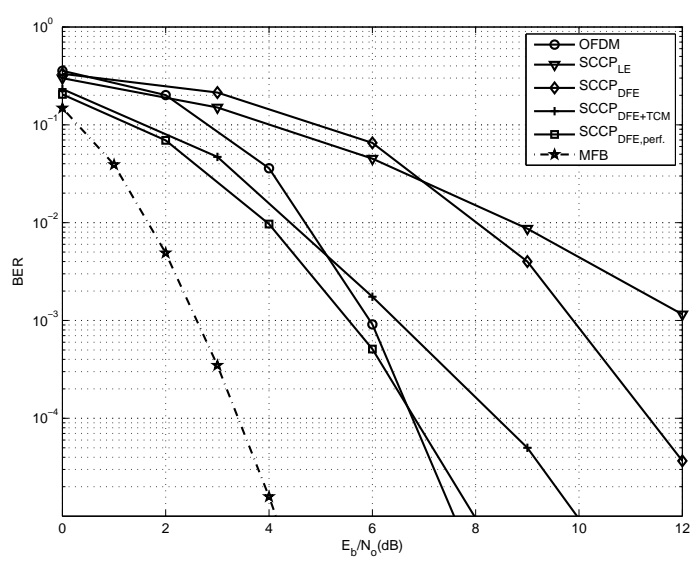

Fig. 11. Comparação de desempenho OFDM (entrelaçador regular com $m=2$ linhas) e SCCP (LE - entreleçador regular com $m=32$ linhas) para o canal estático com função de transferência dada pela eq. (6)

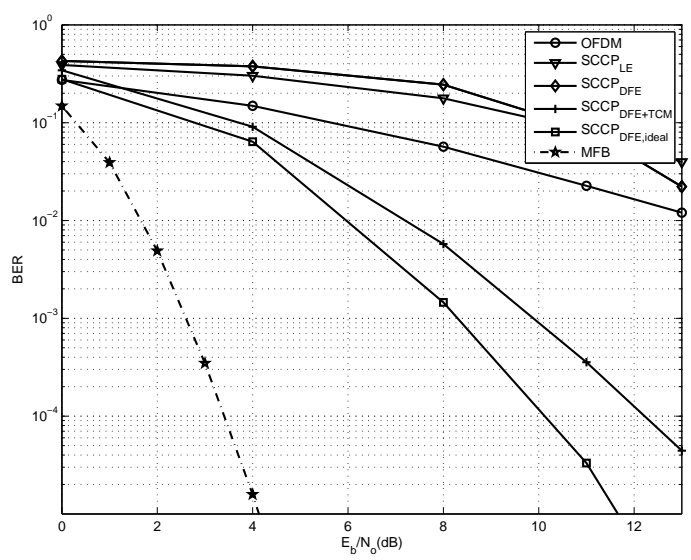

Fig. 12. Comparação de desempenho OFDM (entrelaçador regular com $m=16$ linhas) e SCCP (LE - entreleçador regular com $m=32$ linhas) para o canal estático com função de transferência dada pela eq. (8)

Para tentar responder a essa pergunta, consideremos o canal Proakis C [15] com função transferência:

$$
H(z)=0,227+0,460 z^{-1}+0,688 z^{-2}+0,460 z^{-3}+0,227 z^{-4}
$$

Uma análise semelhante à realizada para o caso do canal Proakis B, revela que a escolha de entrelaçamento regular com $m=16$ linhas é adequada para o sistema OFDM esse canal.

A Fig. 12 mostra que mesmo em condições ideais de entrelaçamento, o desempenho do OFDM ainda é bem inferior ao do DFE ideal. A partir desse resultado, podemos inferir que existem certas condições de canal que se mostram desfavoráveis ao OFDM.

\section{Conclusões}

Nesse trabalho, comparamos os sistemas SCCP e OFDM implementados com diferentes estruturas de entrelaçamento. No contexto de canais seletivos em frequência com desvanecimento por blocos, percebemos a grande sensibilidade do OFDM em relação à configuração do entrelaçador regular. Ainda nesse contexto de canal, observamos que o OFDM é insensível quanto à escolha dos parâmetros do entrelaçador S-aleatório e do entrelaçador modular. No caso de canais seletivos em frequência estáticos, isso ocorre apenas para o entrelaçador S-aleatório. Todavia, é importante frisar que o desempenho desse entrelaçador é inferior ao obtido com os outros tipos de entrelaçadores quando utilizam os parâmetros ótimos.

Já o SCCP é praticamente invariante em relação ao tipo de entrelaçador utilizado, independente do tipo de canal.

Comparando os sistemas OFDM e SCCP, notamos que no caso de canais seletivos em frequência com desvanecimento, o OFDM adequadamente entrelaçado apresenta desempenho equivalente ao do SCCP equalizado com o DFE ideal. Esse mesmo comportamento é observado no caso de alguns canais estáticos. Entretanto, apresentamos uma situação de canal estático na qual nem mesmo a melhor configuração de entrelaçador para o OFDM foi suficiente para tornar seu desempenho comparável ao do SCCP equalizado com o DFE ideal. Tal diferença de desempenho será estudada mais detalhadamente em trabalhos futuros.

\section{REFERÊNCIAS}

[1] H. Sari, G. Karam, and I. Jeanclaude, "Transmission techniques for digital terrestrial TV broadcasting," IEEE Communications Magazine, vol. 33, no. 2, pp. 100-109, Feb. 1995.

[2] V. Aue, G. P. Fettweis, and R. Valenzuela, "A comparison of the performance of linearly equalized single carrier and coded OFDM over frequency selective fading channels using the random coding technique," in Proc. Int. Conf. Communications (ICC), Atlanta, 1998, vol. 2, pp. 753-757.

[3] Y. P. Lin and S. M. Phoong, "BER minimized OFDM systems with channel independent precoders," Signal Processing, IEEE Transactions on, vol. 51, no. 9, pp. 2369-2380, Sept. 2003.

[4] Zhengdao Wang, Xiaoli Ma, and G.B. Giannakis, "OFDM or singlecarrier block transmissions?," Communications, IEEE Transactions on, vol. 52, no. 3, pp. 380-394, March 2004.

[5] S.K. Wilson and J.M. Cioffi, "A comparison of a single-carrier system using a DFE and a coded OFDM system in a broadcast rayleighfading channel," Information Theory, 1995. Proceedings., 1995 IEEE International Symposium on, pp. 335-, Sep 1995.

[6] F. Pancaldi, G. Vitetta, R. Kalbasi, N. Al-Dhahir, M. Uysal, and H. Mheidat, "Single-carrier frequency domain equalization," IEEE Signal Processing Magazine, vol. 25, pp. 37-56, 2008.

[7] J. Tubbax, L. V. der Perre, M Engels, H. De Man, and M. Moonen, "OFDM versus single carrier: A realistic multi-antenna comparison," EURASIP Journal on Applied Signal Processing, vol. 2004, no. 9, pp. 1275-1287, 2004.

[8] N. Benvenuto and S. Tomasin, "On the comparison between OFDM and single carrier modulation with a DFE using a frequency-domain feedforward filter," IEEE Trans. On Communications, vol. 50, no. 6, June 2002.

[9] D. Falconer, S. L. Ariyavisitakul, A. Benjamin-Seeyar, and B. Edison, "Frequency domain equalization for single-cairrier broadband wireless systems," IEEE Communications Magazine, vol. 40, pp. 58-66, April 2002.

[10] G. Ungerboeck, "Channel coding with multilevel/phase signals," Information Theory, IEEE Transactions on, vol. 28, no. 1, pp. 55-67, Jan 1982.

[11] G. Caire, G. Taricco, and E. Biglieri, "Bit-interleaved coded modulation," Information Theory, IEEE Transactions on, vol. 44, no. 3, pp. 927-946, May 1998.

[12] S. Ramseier, "Shuffling bits in time and frequency: an optimum interleaver for OFDM," in International Conference on Communications - ICC, 2003.

[13] H. R. Sadjadpour, N.J.A. Sloane, M. Salehi, and G. Nebe, "Interleaver design for turbo codes," JSAC-Journal on Selected Areas in Communications, vol. 19, pp. 831-837, 2000.

[14] C. M. Panazio and J. M. T. Romano, "Performance of joint spacetime equalization and decoding techniques for wireless systems," in International Telecommunications Symposium-ITS, Natal, Brazil, 2002.

[15] M. Salehi J. Proakis, Digital Communications, Mc Graw-Hill, 5 edition, 2008. 